\title{
Transient thermal NDT: conception in formulae
}

by V. VAVILOV $(*)$

Tomsk Polytechnic University, Russia, TOMSK, 634028, Savinykh, 3.

\begin{abstract}
Thermal Non Destructive Testing acquired some new aspects during the last decade. Now it essentially concerns the characterization of defects requiring the quantitative estimation of involved parameters. In this paper estimation of TNDT sensitivity using classic solutions of heat transfer theory is discussed.
\end{abstract}

\section{Introduction}

Author has been involved into Russian investigations on thermal NDT (TNDT) since 1973 trying to combine the approaches accepted in heat transfer theory, IR technique, image processing and decision making. The idea of this paper is to provide a set of basic formulae which could generate some methodological hints to researchers.

\section{Basic solutions and TNDT sensitivity estimation}

Thermal NDT includes the following steps: 1) thermal stimulation of an object and creation of signal upon defects; 2) temperature measurement; 3) data processing; 4) decision making. Each step deserves the particular discussion and below we make accent onto heat transfer basics. Here we shall deal with $1 \mathrm{D}$ analytical solutions which are widely used in practice.

Table 1 contains the majority of classic solutions adapted from [1]. Notice that expressions for square-pulse heating are given using the Duhamel's theorem in the simplest form: $T\left(\tau, \tau_{h}\right)=T(\tau)-T\left(\tau-\tau_{h}\right)$ and solution for Dirac-pulse heating of non-adiabatic plate (problem 12) is obtained by derivating the corresponding step-function expression (problem 7). Table 2 contains two adapted solutions for multi-layer plates. They represent the independent interest for TNDT theory but methodology discussed below could be applied to them too. (Notice that specification in Tables 1,2 corresponds to the generally accepted).

It is obvious that TNDT problems involve temperature functions which depend on the following parameters: 1) coordinates; 2) time; 3) host specimen size and thermal properties; 4) defect size and thermal properties; 5) heat exchange parameters. For instance, in a case of 3D problem (non-adiabatic conditions, uniform heating), one may count up to 17 parameters in the mentioned set. Below we take as an example 1D solution for a plate heated with Dirac pulse (problem 11 in Table 1 ). In this case surface temperature depends on five parameters: $T(W, \alpha, K, L, \tau)$ time $\tau$ is often expressed as Fourier number $F_{0}=\alpha \tau / L^{2}$.

The following obvious expression can be related to this solution:

$$
\Delta T=\frac{\partial T}{\partial W} \Delta W+\frac{\partial T}{\partial \alpha} \Delta \alpha+\frac{\partial T}{\partial K} \Delta K+\frac{\partial T}{\partial L} \Delta L+\frac{\partial T}{\partial \tau} \Delta \tau
$$

Notice that time is here regarded as common measured parameter which could be considered for thermal characterization. This approach is used now in thermal tomography, effusivity measurement etc. 


\section{http://dx.doi.org/10.21611/qirt.1992.035}

We do not discuss the $W$ parameter which influences temperature as trivial linear function. As an example of $W$ measurement we refer reader to the measurement of laser energy distribution by using the target irradiated with laser and monitored with IR imager.

We introduce here the so called amplitude and temporal parameters which depend in their turn on the diffusivity $\alpha$, conductivity $K$ and plate's thickness $L$. It means that we could characterize the specimen's properties using temperature directly or we could connect these properties to specific heat transfer times (e.g. well known half-rise time $\tau_{0.5}$ which is specified on the rear surface and used in Parker's method for $\alpha$ measurement). As an example we propose to evaluate the TNDT sensitivity with minimum variation of diffusivity or plate's thickness which could be reliably measured on the noise background. Another parameters could be considered too (e.g. effusivity). General expressions are:

$$
\Delta T=\Delta \alpha \frac{\partial T}{\partial \alpha}+\Delta \tau \frac{\partial T}{\partial \tau} ; \quad \Delta T=\Delta L \frac{\partial T}{\partial L}+\Delta \tau \frac{\partial T}{\partial \tau}
$$

If TNDT sensitivity is evaluated by amplitude, relative detectable variations of $L$ and $\alpha$ are:

$$
\frac{\Delta \alpha}{\alpha}=\frac{\Delta T}{\alpha} / \frac{\partial T}{\partial \alpha} ; \quad \frac{\Delta L}{L}=\frac{\Delta T}{L} / \frac{\partial T}{\partial L}
$$

For temporal parameter we could obtain respectively:

$$
\frac{\Delta \alpha}{\alpha}=-\frac{\Delta \tau}{\alpha} \frac{\partial T}{\partial \tau} / \frac{\partial T}{\partial \alpha} ; \quad \frac{\partial L}{L}=-\frac{\Delta \tau}{L} \frac{\partial T}{\partial \tau} / \frac{\partial T}{\partial L}
$$

Ideal sensitivity of any TNDT device is limited by apparatus noise, i.e. by temperature resolution $\Delta T_{\text {res }}$ in amplitude measurements and by temporal resolution $\Delta \tau_{\text {res }}$ in time measurements. It is important to underline that temperature measurements are primary and temporal measurements are secondary depending on $\Delta T_{r e s}$ value which is determined in its turn by detector's noise. In modern IR imagers the $\Delta T_{\text {res }}$ value is about $0.1^{\circ} \mathrm{C}$.

Practical TNDT sensitivity is limited by structural noise which is determined by tested object. It could be characterized with noise contrasts $A_{n}^{T}$ and $A_{n}^{\tau}$ where superscripts $T$ and $\tau$ specify temperature and time measurements respectively. Minimum value of $A_{n}^{T}$ was reported in order of $2 \%$ (for black-painted surfaces) and rough estimation of minimum $A_{n}^{\tau}$ value is in a range of $0.1-0.2 \%[3]$.

\section{TNDT sensitivity is limited by apparatus noise}

Applying equations (3) and (4) to any solution in Tables 1 and 2 it is possible to obtain the sensitivity equations which could be analyzed for minimums of $\Delta \alpha, \Delta K$ and $\Delta L$ values in order to optimize the experimental arrangement. Examples of this methodology for heating of a plate and semi-infinite body are discussed in [3]. In our example (problem 11 in Table 1) we shall consider the heated surface only. If measurements are limited by temperature resolution, corresponding expressions are as follows:

$$
\frac{\Delta \alpha / \alpha}{\Delta T_{r \theta S} / T_{m}}=\left|\frac{1}{\theta-F}\right| ; \quad \frac{\Delta L / L}{\Delta T_{r e s} / T_{m}}=\left|\frac{1}{\theta-2 F}\right|
$$




$$
\theta=1+\sum_{n=1}^{\infty} \exp \left(-n^{2} \pi^{2} F o\right) ; \quad F=2 \pi^{2} F o \sum_{n=1}^{\infty} n^{2} \exp \left(-n^{2} \pi^{2} F o\right) ; \quad T_{m}=W \alpha / K L
$$

In equation (5) we do not specify amplitude and time parameters because they produce the same results because of reasons mentioned above. In order to increase sensitivity in this case we need to enhance first of all $W$ value or use equipment with better temperature resolution. Functions $\frac{\Delta \alpha / \alpha}{\Delta T_{\text {res }} / T_{m}}$ and $\frac{\Delta L / L}{\Delta T_{\text {res }} / T_{m}}$ versus Fo are discussed in [3].

It is interesting that $\frac{\Delta \alpha / \alpha}{\Delta T_{\text {res }} / T_{m}}$ function has maximum at $F o \approx 0.2$ where the worst sensitivity is $\Delta \alpha / \alpha \approx 1.3\left(\Delta T_{\text {res }} / T_{m}\right)$ i.e. $\Delta \alpha / \alpha \approx 1.3 \%$ if $T_{m}=10.0^{\circ} \mathrm{C}$ and $T_{\text {res }}=0.1^{\circ} \mathrm{C}$. The same point ( $F O \approx 0.2$ ) provides the best condition for thickness measurement: $\Delta L / L \approx$ $0.5\left(\Delta T_{\text {res }} / T_{m}\right)$ i.e. about $0.5 \%$ for the example above. Practical recommendation from this abstract consideration is to measure diffusivity immediately after heating and measure thickness at time $F_{O} \approx 0.2$.

\section{TNDT sensitivity is limited by structural noise}

Multiplicative structural noise could not be suppressed by enhancing $\left(\Delta T_{\text {res }} / T_{m}\right)$ value. In this case, amplitude and time parameters could provide different results with time parameters being more resistant against noise. Corresponding equations for diffusivity measuremens are:

$$
\left\langle\frac{\Delta \alpha / \alpha}{A_{n}^{T}}\right\rangle^{T}=\frac{\theta}{\theta-F} ; \quad\left\langle\frac{\Delta \alpha / \alpha}{A_{n}^{\tau}}\right\rangle^{\tau}=\frac{F}{\theta-F}
$$

Detail analysis of equations (6) is done in [3]. Here we just indicate that TNDT sensitivity is improving to the end of process up to values of about $\Delta \alpha / \alpha \approx 0.05-0.1 \%$ and $\Delta \alpha / \alpha \approx 2 \%$ for time and amplitude parameters respectively. It is worth to say that principal limit of sensitivity is given by equations (5). If equations (6) provide better results it just means that temperature or temporal contrasts which are necessary for these results could not be achieved because of limited $\Delta T_{\text {res }}$ value. For instance, theoretically very long measurement time could promise high sensitivity to diffusivity variations but in practice the corresponding signal will be either lost on the noise background (non uniform convection, external radiation, etc.) or specimen's temperature itself will be too low for reliable measurement.

\section{Conclusion}

Basic solutions of heat transfer problems contain the hidden information which is usually omitted in classic research but is of practical interest in thermal characterization of solids.

\section{REFERENCES}

[1] CARSLAW (H.S.) and JAEGER (T.S.). - Conduction of heat in solids. Oxford University Press, Oxford, 1959, U.K. p. 580.

[2] BALAGEAS (D.L.), KRAPEZ (J.C.) and CIELO (P.). - Pulsed photothermal modeling of layered materials. J. Appl. Phys. 59 (2), 15 Jan., 1986, p. 348-357.

[3] VAVILOV (V.P.). - Informativity of thermal fields in the active thermal NDT problems. Sov. J. NDT (Defectoscopia), 1987, №.3, p. 67-77. 
http://dx.doi.org/10.21611/qirt.1992.035

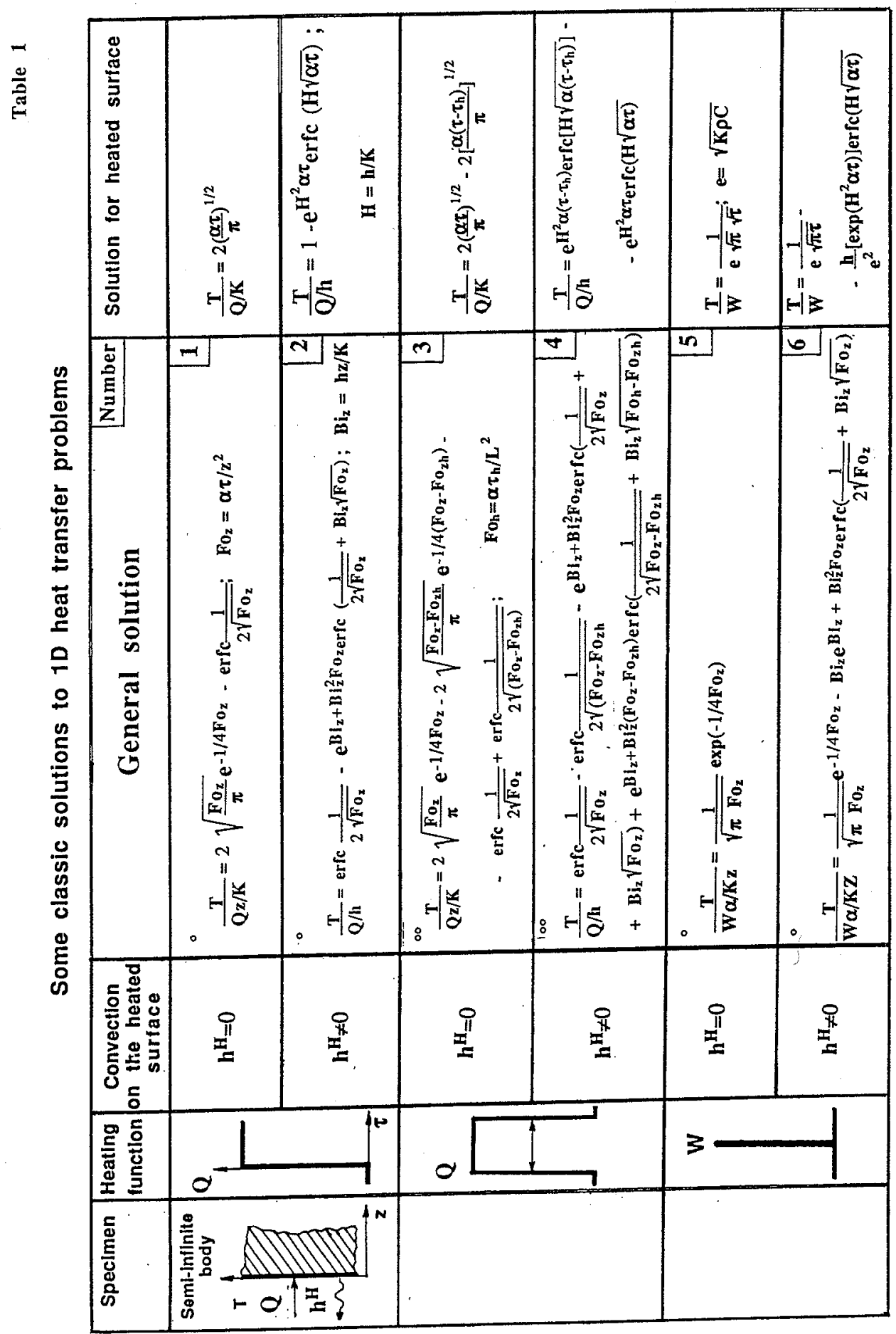


http://dx.doi.org/10.21611/qirt.1992.035

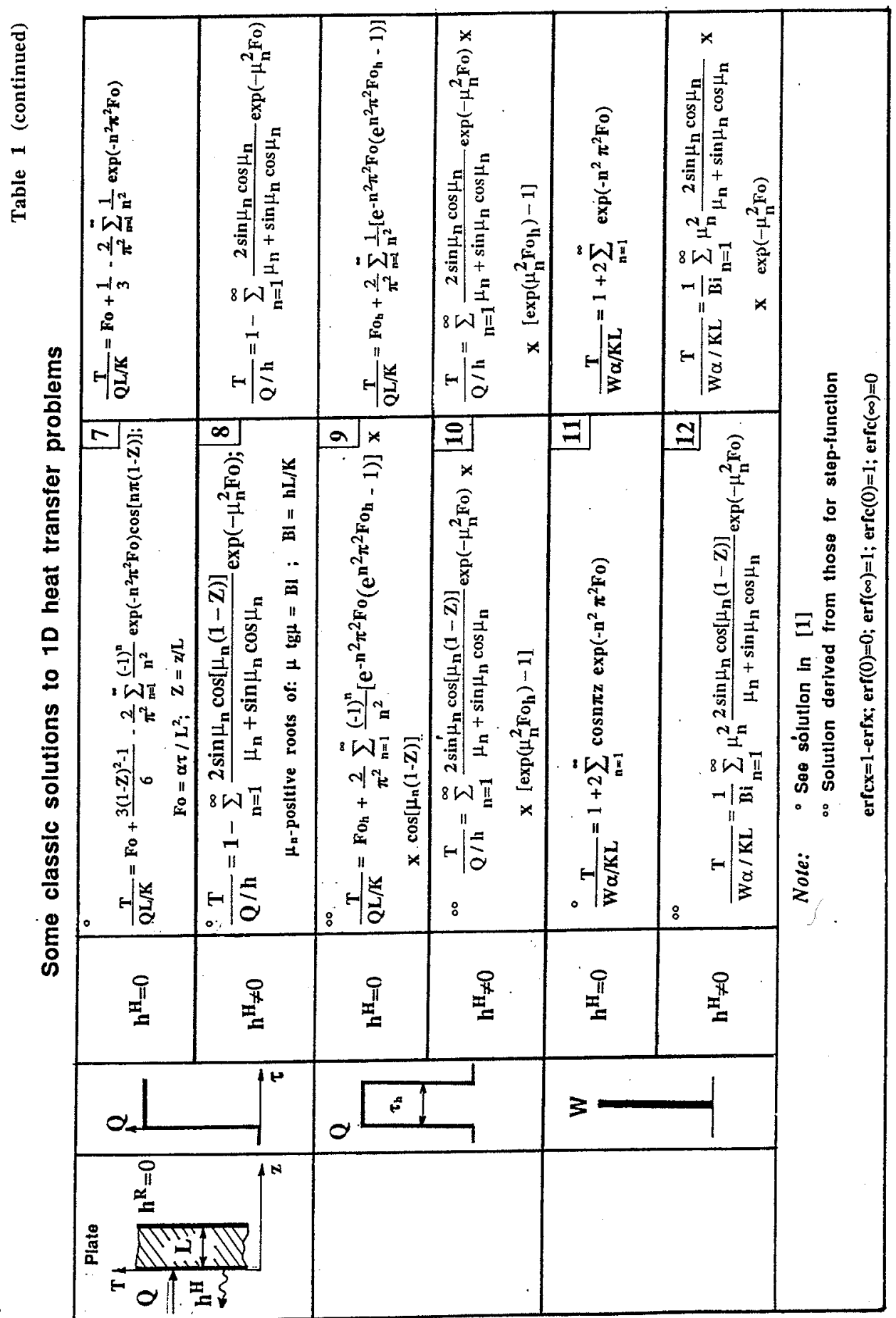


http://dx.doi.org/10.21611/qirt.1992.035

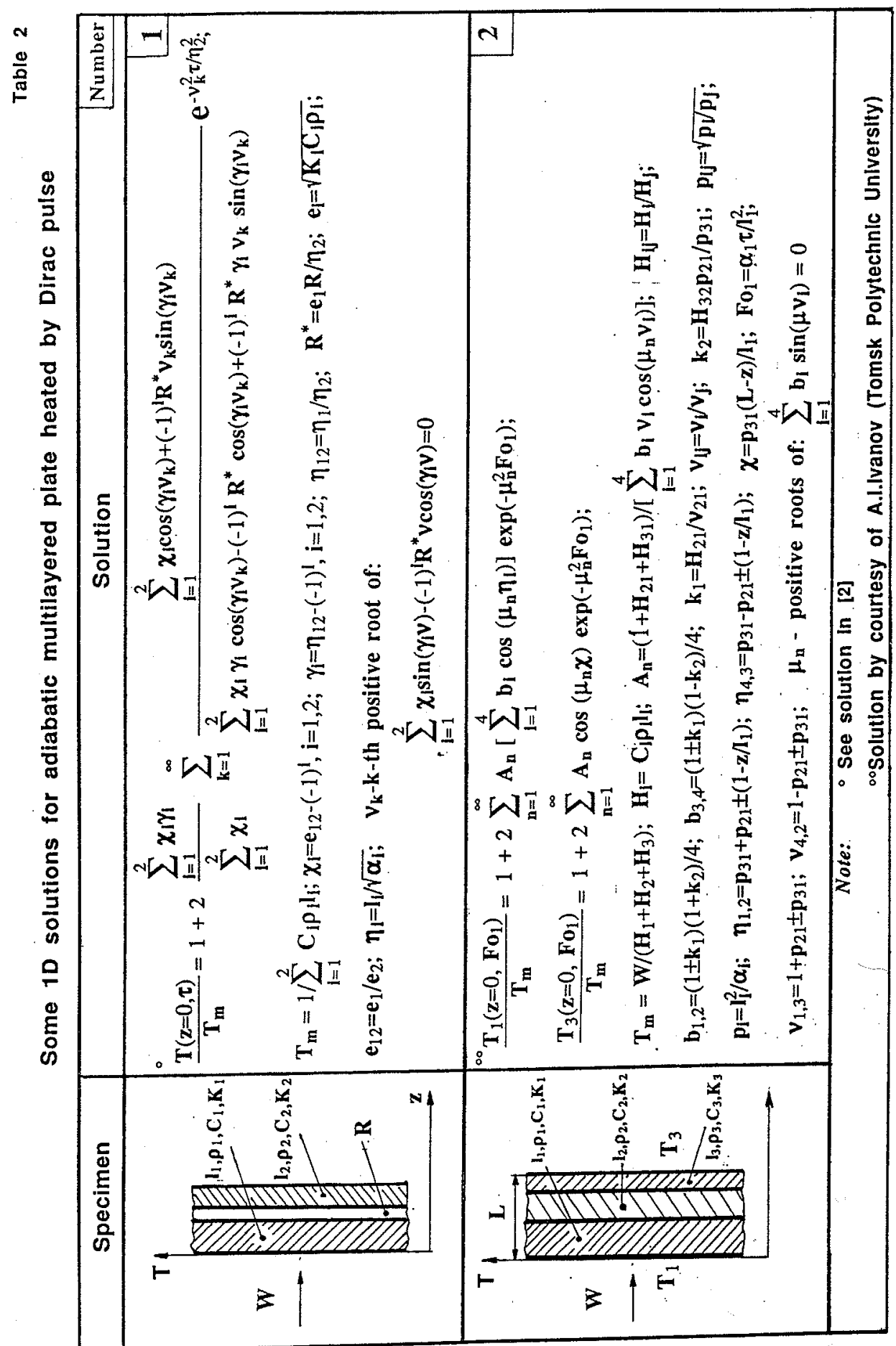

